Research Paper

\title{
CDKN3 expression is an independent prognostic factor and associated with advanced tumor stage in nasopharyngeal carcinoma
}

\author{
Shih-Lun Chang1,2, Tzu-Ju Chen ${ }^{2,3}$, Ying-En Lee ${ }^{4}$, Sung-Wei Lee ${ }^{5}$, Li-Ching Lin' ${ }^{6}$, Hong-Lin He ${ }^{3, 凹}$ \\ 1. Department of Otolaryngology, Chi Mei Medical Center, Tainan, Taiwan \\ 2. Department of Optometry, Chung Hwa University of Medical Technology, Tainan, Taiwan \\ 3. Department of Pathology, Chi-Mei Medical Center, Tainan, Taiwan \\ 4. Department of Anesthesiology, Kaohsiung Chang Gung Memorial Hospital and Chang Gung University College of Medicine, Kaohsiung, Taiwan \\ 5. Department of Radiation Oncology, Chi Mei Medical Center, Liouying, Tainan, Taiwan \\ 6. Department of Radiation Oncology, Chi Mei Medical Center, Tainan, Taiwan \\ $\triangle$ Corresponding author: Hong-Lin He, MD, PhD. Department of Pathology, Chi-Mei Medical Center, Tainan, Taiwan. E-mail: baltic1023@gmail.com. Tel: \\ +886-6-2812811 ext. 53694; Fax: +886-6-2511235 \\ (C) Ivyspring International Publisher. This is an open access article distributed under the terms of the Creative Commons Attribution (CC BY-NC) license \\ (https://creativecommons.org/licenses/by-nc/4.0/). See http://ivyspring.com/terms for full terms and conditions.
}

Received: 2018.01.21; Accepted: 2018.05.27; Published: 2018.06.14

\begin{abstract}
Background: Through data mining from the public transcriptome of NPC, cyclin-dependent kinase inhibitor 3 (CDKN3) was identified as a significantly upregulated gene in NPC. CDKN3 functions as a key factor in cell cycle regulation. This study was aimed to investigate the expression of CDKN3 in NPC tissues and its prognostic significance.

Methods: Immunohistochemistry was performed for 124 NPC patients to assess the protein expression of CDKN3. The stainings of CDKN3 were scored by using $\mathrm{H}$-score method. The relationships between CDKN3 expression status and clinicopathological parameters, disease-specific survival (DSS), distant metastasis-free survival (DMeFS), and local recurrence-free survival (LRFS) were statistically analyzed.

Results: High expression of CDKN3 was significantly associated with higher primary nodal status $(P=0.030)$ and higher TNM stage $(P=0.019)$. In univariate analysis, high expression of CDKN3 predicted worse DSS $(P<0.0001)$, DMeFS $(P<0.0001)$, and LRFS $(P<0.0001)$. In multivariate analysis, CDKN3 overexpression still acted as an independent prognostic factor for worse DSS $(P<0.001$; hazard ratio $[\mathrm{HR}]=11.999,95 \% \mathrm{Cl}$ : 5.378-26.771), DMeFS $(P<0.001 ; \mathrm{HR}=15.069,95 \% \mathrm{Cl}$ : 5.884-38.592), and LRFS $(P<0.001 ; \mathrm{HR}=5.000,95 \% \mathrm{Cl}$ : 2.312-10.815).

Conclusion: High expression of CDKN3 was an independent negative prognostic factor for NPC and was associated with advanced disease status. It might serve as potential therapeutic target and aid in risk stratification for patients with NPC.
\end{abstract}

Key words: CDKN3, nasopharyngeal carcinoma, NPC, cell cycle, transcriptome

\section{Introduction}

Nasopharyngeal carcinoma (NPC) is the most common tumor type arising in the nasopharynx. The occurrence of NPC is caused by a combination of factors, including Epstein-Barr virus (EBV) infection, genetic susceptibility and environmental influences [1]. NPC encompasses three histologic subtypes, including non-keratinizing squamous cell carcinoma, either differentiated or undifferentiated, keratinizing squamous cell carcinoma, and basaloid squamous cell carcinoma [2]. Non-keratinizing squamous cell carcinoma has been found to be closely associated with EBV infection, particularly in endemic areas. In the contrary, EBV infection is much less linked to keratinizing squamous cell carcinoma [3, 4]. With the great advances in diagnostic tools and therapeutic strategies, most of the NPC patients has been under good disease control. However, chemo- or radio-resistance still occurs in a small subset of patients, presenting as local recurrence or distant metastasis. Thus, investigating the molecular 
mechanism that contributes to disease progression is important.

Cell proliferation is an essential mechanism for tumor growth. Dysfunction in cell cycle regulators may aid in carcinogenesis and disease progression. To investigate the role of cell cycle regulation in NPC, we analyzed the expression profiles of NPC transcriptome (GSE12452) from publicly available Gene Expression Omnibus (GEO) database with particular emphasis on genes which are related to the regulation of cyclin-dependent protein kinase activity (GO: 0000079) [5-7]. Cyclin-dependent kinase inhibitor 3 (CDKN3) was identified as the significantly upregulated gene that was associated with advanced disease status. CDKN3 encodes a protein belongs to the dual specificity protein phosphatase family. It functions as dephosphorylating CDK2 kinase at Thr160, and thus prevent the activation of CDK2 [8, 9]. As Thr160 phosphorylation is responsible for the CDK2 activation and cell cycle progression, overexpression of CDKN3 suppresses the G1-S phase progression [10]. Overexpression of CDKN3 has been found in numerous human cancer tissues and cancer cell lines, including hepatocellular carcinoma, lung adenocarcinoma, breast cancer, cervical cancer, ovarian cancer, gastric cancer and renal cancer [11-17]. CDKN3 had an oncogenic role in most of them. Interestingly, CDKN3 has been found to act as a tumor suppressor by controlling mitosis through CDK1 (also known as CDC2 in yeast) signaling axis [18]. In glioblastoma cells, CDKN3 could inhibit cell proliferation and migration via phosphatasedependent inhibition of CDC2. These findings suggested that $\mathrm{CDKN} 3$ may have diverse biological function that can act as either an oncogene or tumor suppressor in different types of cancer cells. However, there is little evidence investigating the definite biologic role of CDKN3 in NPC.

The expression of CDKN3 has never been evaluated in a well-defined cohort of NPC patients. In this study, we tried to figure out the role of CDKN3 in NPC, with particular focus on its prognostic significance in NPC patients. The associations between CDKN3 expression and key clinicopathological parameters were also analyzed.

\section{Material and methods}

\section{Data mining from publicly available NPC transcriptomic dataset}

To find genes associated with the carcinogenesis of NPC, we analyzed the transcriptomic dataset deposited in the Gene Expression Omnibus database (GSE12452), consisting of 31 NPC tissues and 10 non-neoplastic nasopharyngeal mucosal epithelial tissues enriched by laser capture microdissection for cells of interest [7]. We used Nexus Expression 3 software (BioDiscovery) to analyze the raw CEL files of the Affymetrix HUMAN Genome U133 Plus 2.0 microarray platform. All probe sets were analyzed without pre-selection or filtering. We performed supervised comparative analysis to find genes that have significantly different expression, particularly focusing on those related to cyclin-dependent protein kinase activity (GO: 0000079). While comparing tumor versus non-tumor tissues or low-staged versus high-staged cases, those with $P<0.01$ and $\log _{2}$-transformed expression fold change $>0.1$ were selected for further analysis.

\section{Patient characteristics and tumor specimens}

The procurement of formalin-fixed NPC tissue for this study was approved by the Institutional Review Board of Chi Mei Medical Center (IRB10203-001). Between January 1993 and December 2002, 146 NPC patients who received biopsy in Chi Mei Medical Center and with available paraffin-embedded tissue blocks were enrolled in this study. Twenty-two patients who could not fit the selection criteria were excluded, including ten patients diagnosed with systemic disease and another twelve who had not completed a standard course of therapy and/or loss of follow-up. Finally, there were 124 patients enrolled for further analysis. All these patients were free of distant metastasis at initial diagnosis. The histological parameters were reassessed by two pathologists (T.J.C \& H.L.H) who were blind to the clinical information. The tumor staging was based on the $7^{\text {th }}$ American Joint of Cancer Committee (AJCC) system [19]. Of these 124 patients, 114 were under regular monitor after radiotherapy until death or their last appointment. The mean follow-up duration is 67.0 months (range, 3-141). All patients received a complete course of radiotherapy with the daily fractionation of 180 cGy to 200 cGy, five fractions weekly, to achieve a total dose of no less than 7,000 cGy. As a rule, those with stage II-IV disease received at least three cycles of cisplatin-based chemotherapy. However, there was one patient with stage II and four with stage IV disease who received radiotherapy alone due to their poor performance status. Seven patients treated in the earlier phase of this cohort didn't have instantaneous image evaluation after therapy to evaluate treatment response. In total, there were 110 patients with complete response and seven with partial response.

\section{Immunohistochemistry}

Tissue sections of $3-\mu \mathrm{m}$ thickness were cut from paraffin-embedded blocks, and routinely 
deparaffinized with xylene, rehydrated with ethanol, and heated by microwave for antigen retrieval in a 10 $\mathrm{mM}$ citrate buffer $(\mathrm{pH} \mathrm{6)}$ for $7 \mathrm{~min}$. Endogenous peroxidase was quenched by $3 \% \mathrm{H}_{2} \mathrm{O}_{2}$ treatment. Slides were washed with Tris buffered saline for 15 min and then incubated with a primary polyclonal antibody targeting CDKN3 (Rabbit polyclonal, 1:100; Abcam) for one hour. Primary antibodies were detected by using the EnVision system (DAKO, K5001, Carpinteria, CA). The slides were incubated with the secondary antibody for 30 minutes, developed with 3,3-diaminobenzidine for 5 minutes, and then counterstained with hematoxylin. Two pathologists (T.J.C \& H.L.H), blind to the clinical and follow-up information, scored the CDKN3 staining using a multiheaded microscope to reach a consensus on the H-score. The expression of CDKN3 was quantized by using the following equation: $\mathrm{H}$ score $=$ $\sum P i(i+1)$, where $i$ is the intensity (ranging from 0 to 3 ), and $P i$ is the percentage of stained tumor cells varying from $0 \%$ to $100 \%$. The median of $\mathrm{H}$-score served as a cut-off value to sub-classify all cases into low and high expression of CDKN3.

\section{EBER in situ hybridization}

We performed in situ hybridization for the EBV-encoded mRNA (EBER) in an autostainer (Bond MAX, Vision BioSystems Ltd, Mount Waverley, Australia) by using a polymer-based detection system (Bond Polymer Refine Detection, Vision BioSystems Ltd) with an EBV specific probe (Bond ISH EBER Probe) and 3,3'-Diaminobenzidine as chromogen.

\section{Statistical analysis}

The statistical significances of differences between CDKN3 expression and various clincopathological parameters were evaluated by Chi-square test. The endpoints for outcome measurement included disease-specific survival (DSS), distant metastasis-free survival (DMeFS), and local recurrence-free survival (LRFS). Above are calculated from the start date of radiotherapy to the date the event developed. These three survival endpoints were calculated by the Kaplan-Meier method and analyzed by the log-rank test. Multivariate analysis was performed by using the Cox proportional hazards model. All tests are two-sided, and $P$ values less than 0.05 were considered statistically significant. Data analysis was performed using SPSS ver. 14 software.

\section{Results}

\section{CDKN3 is the significantly upregulated gene related to cyclin-dependent protein kinase activity in NPC}

To find genes associated with the carcinogenesis and progression of NPC, we analyzed the publicly available transcriptome (GSE12452) deposited in Gene Expression Omnibus (GEO) database, including 31 NPC cases and 10 normal reference samples. We focused on genes related to cyclin-dependent protein kinase activity and found that CDKN3 showed the greatest $\log _{2}$-transformed expression fold change when comparing tumor versus non-tumor $\left(\log _{2}\right.$ ratio $=0.7035$ and $0.9212 ; P=0.0008$ and $<0.0001$, respectively) and high-staged (III-IV) versus low-staged (I-II) tumors ( $\log _{2}$ ratio $=0.5747$ and $0.6007 ; P=0.0046$ and 0.0053 , respectively). (Figure 1, Table 1). Thus, CDKN3 was selected for further study and analysis.

Table 1. Summary of significant differentially expressed genes related to regulation of cyclin-dependent protein kinase activity (GO: 0000079) and associated with tumorigenesis and progression of NPC in the transcriptome of nasopharyngeal carcinoma (GSE12452).

\begin{tabular}{|c|c|c|c|c|c|c|c|}
\hline \multirow[t]{2}{*}{ Probe } & \multicolumn{2}{|c|}{$\begin{array}{l}\text { Comparing tumor to } \\
\text { non-tumor }\end{array}$} & \multicolumn{2}{|c|}{$\begin{array}{l}\text { Comparing AJCC stage III to } \\
\text { AJCC stage I-II }\end{array}$} & \multirow[t]{2}{*}{$\begin{array}{l}\text { Gene } \\
\text { Symbol }\end{array}$} & \multirow[t]{2}{*}{ Gene Name } & \multirow[t]{2}{*}{ Molecular Function } \\
\hline & $\begin{array}{l}\text { Comparison } \\
\log _{2} \text { ratio }\end{array}$ & $\begin{array}{l}\text { Comparison } \\
P \text { value }\end{array}$ & $\begin{array}{l}\text { Comparison } \\
\log _{2} \text { ratio }\end{array}$ & $\begin{array}{l}\text { Comparison } P \\
\text { value }\end{array}$ & & & \\
\hline 1555758_a_at & 0.7035 & 0.0008 & 0.5747 & 0.0046 & CDKN3 & \multirow{2}{*}{$\begin{array}{l}\text { cyclin-dependent kinase } \\
\text { inhibitor } 3 \\
\text { (CDK2-associated dual } \\
\text { specificity phosphatase) }\end{array}$} & \multirow{2}{*}{$\begin{array}{l}\text { hydrolase activity, kinase activity, phosphoprotein } \\
\text { phosphatase activity, phosphoric monoester hydrolase } \\
\text { activity, protein binding, protein tyrosine phosphatase } \\
\text { activity, protein tyrosine/serine/threonine phosphatase } \\
\text { activity }\end{array}$} \\
\hline 209714_s_at & 0.9212 & $<0.0001$ & 0.6007 & 0.0053 & CDKN3 & & \\
\hline
\end{tabular}

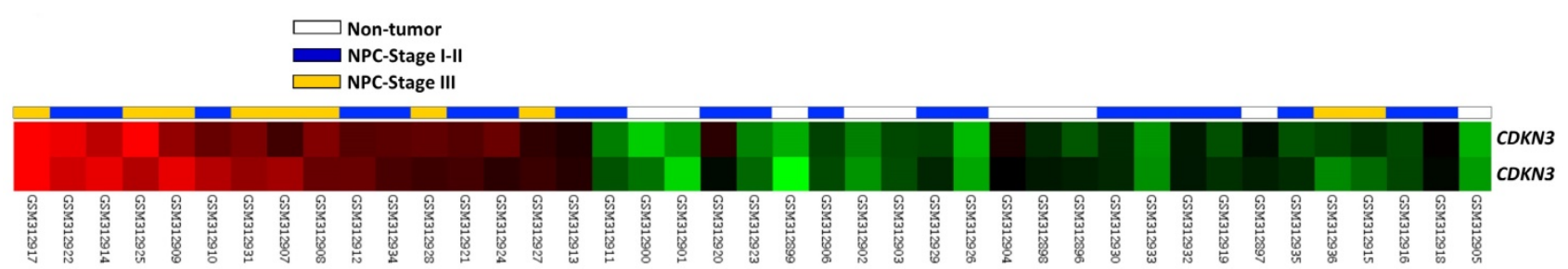

Figure 1. Data mining of public transcriptome of NPC (GSE12452). Comparative analysis of genes related to cyclin-dependent protein kinase activity (GO:0000079) showed that CDKN3 was significantly upregulated in tumor tissues and high-staged cases, when compared with non-tumoral tissues and low-staged cases, respectively. 

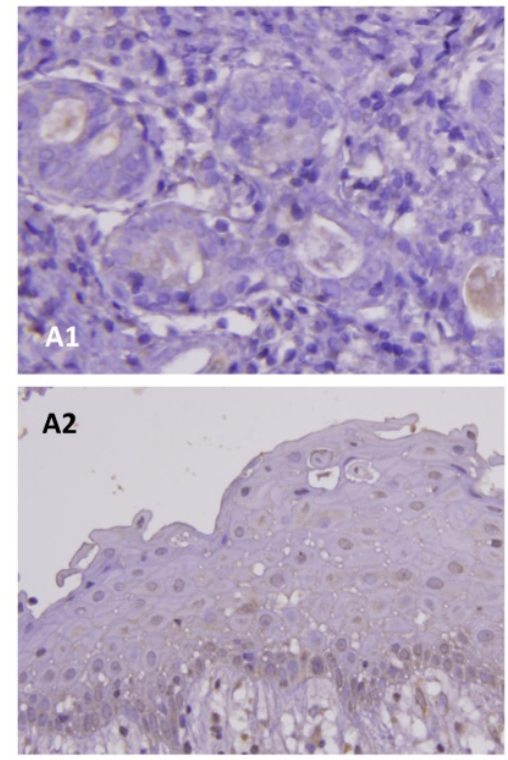

Figure 2. Immunohistochemical study of CDKN3 expression. The protein expression of CDKN3 was not observed in the normal salivary glands (A1) and benign nasopharyngeal mucosa (A2). There were weak and strong cytoplasmic stainings of CDKN3 in representative low-staged (B) and high-staged tumors (C), respectively.

\section{Associations between CDKN3 expression and clinicopathological parameters}

As shown in Table 2, this cohort included 95 males and 29 females with a mean age of 48.6 years (range, 20-83). Clinical stages of the 124 patients were stage I $(n=7)$, II $(n=31)$, III $(n=46)$, and IV $(n=40)$. Histologic types of these patients were keratinizing $(n=5)$, non-keratinizing, differentiated $(n=54)$ and undifferentiated $(n=65)$. All the tumors mentioned above were positive for EBER except for one keratinizing NPC. In the normal salivary glands (Figure 2A1) and nasopharyngeal mucosa (Figure 2A2), there was no expression of CDKN3. There were variable cytoplasmic stainings in the tumor samples with H-scores ranging from 110 to 380. A representative low-staged NPC showed weak cytoplasmic staining of CDKN3 in tumor cells (Figure 2B). Another one representative high-staged NPC showed strong cytoplasmic staining (Figure 2C).

Moreover, we tried to investigate whether there were correlations between CDKN3 expression and clinicopathological parameters. The result reveals that

\section{CDKN3 overexpression is associated with poor survival in NPC}

The mean time of follow up was 67 months from the initial diagnosis (range, 3-141). In addition to T3-4 status, N2-3 status, and AJCC III-IV stage, high expression of CDKN3 was significantly predictive for worse DSS $(P<0.0001$, Figure 3A), DMeFS $(P<0.0001$, Figure 3B), and LRFS $(P<0.0001$, Figure $3 C)$ in univariate analysis (Table 3 ). The CDKN3 expression high expression of CDKN3 was significantly associated with higher primary nodal status $(P=0.030)$ and higher TNM stage $(P=0.019)$ (Table 2).

Table 2. Associations between CDKN3 expression and other important clinicopathological variables.

\begin{tabular}{|c|c|c|c|c|}
\hline \multirow[t]{2}{*}{ Parameters } & \multirow[t]{2}{*}{ Category } & \multicolumn{2}{|c|}{ CDKN3 Exp. } & \multirow[t]{2}{*}{$P$ value } \\
\hline & & Low & High & \\
\hline \multirow[t]{2}{*}{ Gender } & Male & 49 & 46 & 0.524 \\
\hline & Female & 13 & 16 & \\
\hline \multirow[t]{2}{*}{ Age (years) } & $<60$ years & 49 & 49 & 1.000 \\
\hline & $>=60$ years & 13 & 13 & \\
\hline \multirow[t]{2}{*}{ Primary tumor $(\mathrm{T})$} & $\mathrm{T} 1-\mathrm{T} 2$ & 45 & 35 & 0.061 \\
\hline & T3-T4 & 17 & 27 & \\
\hline \multirow[t]{2}{*}{ Nodal status (N) } & N0-N1 & 34 & 22 & $0.030^{*}$ \\
\hline & N2-N3 & 28 & 40 & \\
\hline \multirow[t]{2}{*}{ Stage } & I-II & 25 & 13 & $0.019 *$ \\
\hline & III-IV & 37 & 49 & \\
\hline \multirow[t]{3}{*}{ Histological grade } & Keratinizing & 2 & 3 & 0.192 \\
\hline & Non-keratinizing & 32 & 22 & \\
\hline & Undifferentiated & 28 & 37 & \\
\hline \multirow[t]{2}{*}{ EBER } & Negative & 0 & 1 & 0.315 \\
\hline & Positive & 62 & 61 & \\
\hline
\end{tabular}

*, Statistically significant

and TNM stage were analyzed together in a Cox proportional hazards regression model. In multivariate comparison, high expression of CDKN3 still acted as an independent prognostic factor for shorter DSS $(P<0.001$; hazard ratio $[\mathrm{HR}]=11.999,95 \%$ CI: 5.378-26.771), DMeFS $(P<0.001 ; \mathrm{HR}=15.069,95 \%$ CI: 5.884-38.592), and LRFS $(P<0.001 ; \mathrm{HR}=5.000,95 \%$ CI: 2.312-10.815) (Table 4). 



Figure 3. Survival analysis of NPC patients according to CDKN3 expression by using Kaplan-Meier estimator. The impact of CDKN3 expression on survivals was analyzed by log-rank test. High expression of CDKN3 was significantly associated with worse disease-specific (A), distant metastasis-free (B), and local recurrence-free survival (C).

Table 3. Univariate log-rank analyses.

\begin{tabular}{|c|c|c|c|c|c|c|c|c|}
\hline \multirow[t]{2}{*}{ Parameters } & \multirow[t]{2}{*}{ Category } & \multirow[t]{2}{*}{ No. of case } & \multicolumn{2}{|l|}{ DSS } & \multicolumn{2}{|l|}{ DMeFS } & \multicolumn{2}{|l|}{ LRFS } \\
\hline & & & No. of event & $P$ value & No. of event & $P$ value & No. of event & $P$ value \\
\hline \multirow[t]{2}{*}{ Gender } & Male & 95 & 45 & 0.7870 & 38 & 0.6128 & 30 & 0.3240 \\
\hline & Female & 29 & 14 & & 11 & & 7 & \\
\hline \multirow[t]{2}{*}{ Age (years) } & $<60$ years & 98 & 48 & 0.8600 & 42 & 0.3091 & 29 & 0.8206 \\
\hline & $>=60$ years & 26 & 11 & & 7 & & 8 & \\
\hline \multirow[t]{2}{*}{ Primary tumor $(\mathrm{T})$} & $\mathrm{T} 1-\mathrm{T} 2$ & 80 & 32 & $0.0289 *$ & 25 & $0.0085^{*}$ & 19 & $0.0180^{*}$ \\
\hline & $\mathrm{T} 3-\mathrm{T} 4$ & 44 & 27 & & 24 & & 18 & \\
\hline \multirow[t]{2}{*}{ Nodal status (N) } & N0-N1 & 56 & 18 & $0.0008^{*}$ & 17 & $0.0132^{*}$ & 12 & $0.0160^{*}$ \\
\hline & N2-N3 & 68 & 41 & & 32 & & 25 & \\
\hline \multirow[t]{2}{*}{ Stage } & I-II & 38 & 10 & $0.0020^{*}$ & 9 & $0.0072^{*}$ & 5 & $0.0026^{*}$ \\
\hline & III-IV & 86 & 49 & & 40 & & 32 & \\
\hline \multirow[t]{2}{*}{ Histological grade } & Keratinizing/Non-keratinizing & 47 & 20 & 0.1980 & 17 & 0.2753 & 15 & 0.9521 \\
\hline & Undifferentiated & 77 & 39 & & 32 & & 22 & \\
\hline \multirow[t]{2}{*}{ EBER } & Negative & 1 & 1 & 0.0577 & 1 & 0.0937 & 0 & 0.7305 \\
\hline & Positive & 123 & 58 & & 48 & & 37 & \\
\hline \multirow[t]{2}{*}{ CDKN3 Exp. } & Low Exp. (H-score<median) & 62 & 7 & $<0.0001^{*}$ & 5 & $<0.0001^{*}$ & 9 & $<0.0001^{*}$ \\
\hline & High Exp. (H-score>=median) & 62 & 52 & & 44 & & 28 & \\
\hline
\end{tabular}

*, Statistically significant; DSS, disease-specific survival; DMeFS, distal metastasis-free Survival; LRFS, local recurrence-free survival

Table 4. Multivariate survival analyses

\begin{tabular}{|c|c|c|c|c|c|c|c|c|c|c|}
\hline \multirow[t]{2}{*}{ Parameter } & \multirow[t]{2}{*}{ Category } & \multicolumn{3}{|l|}{ DSS } & \multicolumn{3}{|c|}{ DMeFS } & \multicolumn{3}{|l|}{ LRFS } \\
\hline & & $\overline{\text { HR }}$ & $95 \%$ CI & $P$ value & HR & $95 \% \mathrm{CI}$ & $P$ value & HR & $95 \% \mathrm{CI}$ & $P$ value \\
\hline \multirow[t]{2}{*}{ Stage } & I-II & 1 & - & 0.121 & 1 & - & 0.183 & 1 & & $0.037^{*}$ \\
\hline & III-IV & 1.725 & $0.867-3.431$ & & 1.642 & $0.791-3.406$ & & 2.766 & 1.064-7.191 & \\
\hline \multirow[t]{2}{*}{ CDKN3 Exp. } & Low Exp. & 1 & - & $<0.001^{*}$ & 1 & - & $<0.001^{*}$ & 1 & - & $<0.001^{*}$ \\
\hline & High Exp. & 11.999 & $5.378-26.771$ & & 15.069 & 5.884-38.592 & & 5.000 & $2.312-10.815$ & \\
\hline
\end{tabular}

*, Statistically significant; DSS, disease-specific survival; DMeFS, distal metastasis-free Survival; LRFS, local recurrence-free survival; HR, hazard ratio; CI, confidence interval

\section{Discussion}

In the present study, our result disclosed that high expression of CDKN3 was observed in human NPC tissue, and CDKN3 overexpression was significantly associated with advanced nodal status and advanced tumor staging. Moreover, high expression of CDKN3 was an independent negative prognostic factor for DSS, DMeFS and LRFS. These findings suggested that there is an oncogenic role of CDKN3 in NPC. In human gastric cancer cells
SGC-7901, knockdown of CDKN3 significantly suppressed cell proliferation, migration, invasion and adhesion abilities. Also, depletion of CDKN3 expression induced SGC-7901 cell apoptosis. In patients with gastric cancer, high expression levels of CDKN3 were significantly associated with advanced clinical staging and recurrence. Overall survival was significantly shorter in patient with high CDKN3 expression compared with those with low expression [16]. In ovarian cancer, there was similar finding that knockdown of CDKN3 expression significantly 
inhibited cell proliferation in OVCAR3 cell line [15]. Additionally, in patients with ovarian cancer, high expression of CDKN3 was significantly associated with worse overall survival and disease-free survival. In breast cancer cells, silencing of CDKN3 in MCF-7 and BT474 cell lines induced G1 phase cell cycle arrest, increased cell apoptosis, and inhibited cell migration. Moreover, knockdown of CDKN3 suppressed proliferating cell nuclear antigen (PCNA), Bcl-2, vimentin and Ras homolog gene family, member A (RhoA) expression, and increased Bax expression, which suggested that CDKN3 acts as an oncogene in breast cancer [12]. In a recent study, CDKN3 was found to have an oncogenic role in NPC [20]. The author found that downregulation of CDKN3 inhibited cell proliferation, suppressed cell invasion, induced cell cycle arrest activated apoptosis and increased radiosensitivity. Moreover, silencing of p27 significantly suppressed the effect of the knockdown of CDKN3 on these biological behaviors. The expression of CDKN3 was inversely associated with that of p27 in NPC patients. This finding indicated that CDKN3 had an oncogenic role in NPC by targeting $\mathrm{p} 27$.

Interestingly, some previous studies suggested that CDKN3 has a potential tumor suppressor role. In hepatocellular carcinoma cells, knockdown of CDKN3 increased colony formation capacity and cisplatin tolerance [21]. The authors also found that depletion of CDKN3 activates the AKT/p53/p21 signaling pathway. Additionally, in Bcr-Abl-mediated leukemogenesis, CDKN3 also acted as a tumor suppressor. Overexpression of CDKN3 increased the susceptibility of the K562 leukemic cells to imanitib-induced apoptosis and suppressed in vivo K562 xenografted tumor growth. Conversely, depletion of CDKN3 expression resulted in resistance to imatinib-induced apoptosis in the leukemic cells and increased the tumor growth of K562 xenograft in mice. Reduced CDK2 dephosphorylation and delayed G1/S transition were also observed in CDKN3-overexpressing K562 leukemic cells [22]. This study also demonstrated that CDKN3 contributes to suppressing CDK2-dependent XIAP expression. $\mathrm{XIAP}$, an anti-apoptotic factor regulated by CDK2, has been found to play a critical role in regulating cell survival [23-25]. Thus, CDKN3 may negatively regulate cell survival by dephosphorylating CDK2, thereby decreasing CDK2-dependent XIAP expression in leukemic cells.

In regard to the perception of $C D K N 3$ as a tumor suppressor gene in human cancer, some possible assumptions for explaining CDKN3 overexpression in tumors are mutation or alternative splicing leading to aberrant CDKN3 transcripts that encode dominant-negative products of CDKN3. However, CDKN3 mutation or copy number alternation is rare in human cancers and aberrant CDKN3 transcripts occur infrequently and usually at lower levels [13]. These findings cannot explain overexpression of CDKN3 in a wide variety of tumors and question the perception of CDKN3 as a tumor suppressor. CDKN3 has the ability to bind to CDK1 and CDK2 and causes dephosphorylation of the activating residues, leading to suppression of the CDK activities [9, 10]. CDK-driven cell cycle is a major event for proliferation of cancer cells. Though CDKN3 functions as negative regulator of CDK1 and CDK2, we still cannot regard CDKN3 as a tumor suppressor since there are dynamic changes of CDK activities along the cell cycle. The regulation of cell cycle depends not only CDK activation but also CDK deactivation. The final effect of CDKN3 in cell proliferation, either stimulating or suppressing, in different types of cancers cannot be explained by its regulation in CDK alone. In our study, our result supported that CDKN3 has an oncogenic role, rather than acted as a tumor suppressor. More basic researches are needed to clarify the diverse biological function in different kinds of cancer.

In this study, we found that overexpression of CDKN3 was significantly associated with advanced disease status and worse survival, including DSS, DMeFS and LRFS. Our result supported the oncogenic role of CDKN3 in NPC. The molecular mechanism about the diverse functional properties of CDKN3 in different cancer needs to be further studied. In NPC, high expression of CDKN3 is a negative prognostic factor and may serve as a potential therapeutic target.

\section{Abbreviations}

NPC: Nasopharyngeal carcinoma; EBV: Epstein-Barr virus; GEO: Gene Expression Omnibus, CDKN3: cyclin-dependent kinase inhibitor 3; AJCC: American Joint of Cancer Committee; EBER: EBV-encoded mRNA; DSS: disease-specific survival; DMeFS: distant metastasis-free survival; LRFS: local recurrence-free survival; PCNA: proliferating cell nuclear antigen; RhoA: Ras homolog gene family, member A.

\section{Acknowledgements}

This study was supported by a grant from the Ministry of Health and Welfare (MOHW103-TD-B111-05). The authors also thank Biobank and Translational Research Laboratory of Human Cancers at Chi Mei Medical Center for providing the tumor samples and technical supports. 


\section{Competing Interests}

The authors have declared that no competing interest exists.

\section{References}

1. Wei WI, Sham JS. Nasopharyngeal carcinoma. Lancet. 2005; 365: 2041-54.

2. El-Naggar AK, Chan JKC, Grandis JR, Takata T, Slootweg PJ. WHO Classification of Head and Neck Tumours. WHO/IARC Classification of Tumours, 4th Edition.

3. Marks JE, Phillips JL, Menck HR. The National Cancer Data Base report on the relationship of race and national origin to the histology of nasopharyngeal carcinoma. Cancer. 1998; 83: 582-8.

4. Niedobitek G, Hansmann ML, Herbst H, Young LS, Dienemann D, Hartmann CA, et al. Epstein-Barr virus and carcinomas: undifferentiated carcinomas but not squamous cell carcinomas of the nasopharynx are regularly associated with the virus. J Pathol. 1991; 165: 17-24.

5. Dodd LE, Sengupta S, Chen IH, den Boon JA, Cheng YJ, Westra W, et al. Genes involved in DNA repair and nitrosamine metabolism and those located on chromosome 14q32 are dysregulated in nasopharyngeal carcinoma. Cancer Epidemiol Biomarkers Prev. 2006; 15: 2216-25.

6. Hsu WL, Tse KP, Liang S, Chien YC, Su WH, Yu KJ, et al. Evaluation of human leukocyte antigen-A (HLA-A), other non-HLA markers on chromosome 6p21 and risk of nasopharyngeal carcinoma. PLoS One. 2012; 7: e42767.

7. Sengupta S, den Boon JA, Chen IH, Newton MA, Dahl DB, Chen M, et al. Genome-wide expression profiling reveals EBV-associated inhibition of MHC class I expression in nasopharyngeal carcinoma. Cancer Res. 2006; 66: 7999-8006.

8. Gyuris J, Golemis E, Chertkov H, Brent R. Cdi1, a human G1 and S phase protein phosphatase that associates with Cdk2. Cell. 1993; 75: 791-803.

9. Hannon GJ, Casso D, Beach D. KAP: a dual specificity phosphatase that interacts with cyclin-dependent kinases. Proc Natl Acad Sci U S A. 1994; 91 : 1731-5.

10. Poon RY, Hunter T. Dephosphorylation of Cdk2 Thr160 by the cyclin-dependent kinase-interacting phosphatase KAP in the absence of cyclin. Science. 1995; 270: 90-3.

11. Barron EV, Roman-Bassaure E, Sanchez-Sandoval AL, Espinosa AM, Guardado-Estrada M, Medina I, et al. CDKN3 mRNA as a Biomarker for Survival and Therapeutic Target in Cervical Cancer. PLoS One. 2015; 10: e0137397.

12. Deng $\mathrm{M}$, Wang J, Chen $\mathrm{Y}$, Zhang L, Xie G, Liu O, et al. Silencing cyclin-dependent kinase inhibitor 3 inhibits the migration of breast cancer cell lines. Molecular Medicine Reports. 2016; 14: 1523-30.

13. Fan C, Chen L, Huang Q, Shen T, Welsh EA, Teer JK, et al. Overexpression of major CDKN3 transcripts is associated with poor survival in lung adenocarcinoma. Br J Cancer. 2015; 113: 1735-43.

14. Lai MW, Chen TC, Pang ST, Yeh CT. Overexpression of cyclin-dependent kinase-associated protein phosphatase enhances cell proliferation in renal cancer cells. Urol Oncol. 2012; 30: 871-8.

15. Li T, Xue H, Guo Y, Guo K. CDKN3 is an independent prognostic factor and promotes ovarian carcinoma cell proliferation in ovarian cancer. Oncol Rep. 2014; 31: 1825-31.

16. Li Y, Ji S, Fu LY, Jiang T, Wu D, Meng FD. Knockdown of Cyclin-Dependent Kinase Inhibitor 3 Inhibits Proliferation and Invasion in Human Gastric Cancer Cells. Oncol Res. 2017; 25: 721-31.

17. Xing C, Xie H, Zhou L, Zhou W, Zhang W, Ding S, et al. Cyclin-dependent kinase inhibitor 3 is overexpressed in hepatocellular carcinoma and promotes tumor cell proliferation. Biochem Biophys Res Commun. 2012; 420: 29-35.

18. Nalepa G, Barnholtz-Sloan J, Enzor R, Dey D, He Y, Gehlhausen JR, et al. The tumor suppressor CDKN3 controls mitosis. J Cell Biol. 2013; 201: 997-1012.

19. Edge SB, Compton CC. The American Joint Committee on Cancer: the 7th edition of the AJCC cancer staging manual and the future of TNM. Ann Surg Oncol. 2010; 17: 1471-4.

20. Wang $\mathrm{H}$, Chen $\mathrm{H}$, Zhou H, Yu W, Lu Z. Cyclin-Dependent Kinase Inhibitor 3 Promotes Cancer Cell Proliferation and Tumorigenesis in Nasopharyngeal Carcinoma by Targeting p27. Oncol Res. 2017; 25: 1431-40.

21. Dai W, Miao H, Fang S, Fang T, Chen N, Li M. CDKN3 expression is negatively associated with pathological tumor stage and CDKN3 inhibition promotes cell survival in hepatocellular carcinoma. Mol Med Rep. 2016; 14: 1509-14.

22. Chen $\mathrm{Q}$, Chen $\mathrm{K}$, Guo G, Li F, Chen $\mathrm{C}$, Wang S, et al. A critical role of CDKN3 in Bcr-Abl-mediated tumorigenesis. PLoS One. 2014; 9: e111611.

23. Dong F, Guo W, Zhang L, Wu S, Teraishi F, Davis JJ, et al. Downregulation of XIAP and induction of apoptosis by the synthetic cyclin-dependent kinase inhibitor GW8510 in non-small cell lung cancer cells. Cancer Biol Ther. 2006; 5: $165-70$.

24. Jost PJ, Grabow S, Gray D, McKenzie MD, Nachbur U, Huang DC, et al. XIAP discriminates between type I and type II FAS-induced apoptosis. Nature. 2009; 460: 1035-9.

25. Silva KL, de Souza PS, Nestal de Moraes G, Moellmann-Coelho A, Vasconcelos Fda C, Maia RC. XIAP and P-glycoprotein co-expression is related to imatinib resistance in chronic myeloid leukemia cells. Leuk Res. 2013; 37 : $1350-8$ 